\title{
LXIII. Notice relating to the theory of the equilibrium of fluids
}

\author{
J. Ivory Esq. M.A. F.R.S.
}

To cite this article: J. Ivory Esq. M.A. F.R.S. (1826) LXIII. Notice relating to the theory of the equilibrium of fluids, Philosophical Magazine Series 1, 67:338, 439-442, DOI: 10.1080/14786442608674156

To link to this article: http://dx.doi.org/10.1080/14786442608674156

册 Published online: 10 Aug 2009.

Submit your article to this journal $\pi$

Џ Article views: 2

Q View related articles 5 
Mr. Ivory on the Theory of the Equilibrium of Fluids. 439

have been thus particularized, on the Pheasant's progress in her voyage commencing at Sierra Leone, and terminating at New York, it may be seen, that she was indebted to their aid on the balance of the whole account, and in the direction of her course from port to port, not less than 1600 geographical miles, the whole distance being under 9000 miles; affording a very striking exemplification of the importance of a correct knowledge of the currents of the ocean to persons engaged in its navigation ; and consequently of the value of the information, in the acquisition and arrangement of which Major Rennell has passed the later years of his most useful life. The publication of the charts of the currents in the most frequented parts of the ocean, which he has prepared with his accustomed and well-known indefatigable assiduity, and strict adherence to the evidence of facts, - as soon as he shall deem them sufficiently complete for the public guidance, -will be a most important service rendered to practical navigation.

LXIII. Notice relating to the Theory of the Equilibrium of Fluids. By J. Ivory, Esq. Mi.A. F.R.S.

To the Editor of the Philosophical Magazine and Journal. Sir,

A S I find that what I have written on the equilibrium of $A$ fluids is misrepresented on some occasions, I must beg leave to occupy a small space in your Journal, in stating correctly the points I have endeavoured to establish.

In the Philosophical Transactions for 1824, and in the pages of your Journal, I have treated specially of a homogeneous fluid. The equilibrium in this case, according to what is laid down in all the books, is contained in a single law; namely, that the gravitation at the outer surface be directed inward at right angles to that surface, at the same time that the differential equation is integrable without supposing any relation between the coordinates. That this is a correct account of the received theory I need only cite the Mécanique Céleste, Liv. $1^{\text {me }}$. Nos. 17 and 34 . Liv, $3^{\text {me }}$. No. 22 . No condition is required with respect to the interior of the fluid, and the reason is expressly given. Comme on peut dans l'interieur d'une masse homogene, prendre telles conches que l'on veut, pour couches de densité constante; la seconde des deux conditions precedentes de l'equilibre, est toujours satisfaite, et il suffit pour l'equilibre, que la première soit remplie; c'est-a-dire que la resultante 
sultante de toutes les forces qui animent chaque molécule de la surface cxterieure, soit perpendiculaire à cette surface.-Méc Cél. Liv. $3^{\mathrm{m} e}$. No. 22. Having now described accurately the received theory of the equilibrium of a homogeneous fluid, I have next to add, that $I$ found it defective and insufficient. The defect arises from neglecting to consider the level strata in the interior of the mass. These strata are derived from the equation of the outer surface in the same manner in a homogeneous fluid as in one of variable density. Their mathematical definition is the same in buth cases, although the physical distinction arising from difference of density which abtains in one case is lost in the other. They are not more arbitrary when the fluid is homogeneous than when its density varies from one stratum to another. Viewing the matter in this light, I supplied the defect of the received theory in two different ways: First, by the principle that every level straturm attracts a particle in the inside with equal force in opposite directions: Secondly, by this other principle or theorem, If a homogeneous fluid body revolving about an axis, be in equilibrio by the attraction of its particles in the inverse proportion of the square of the distance; any other mass of the same fuid having a similar figure and revolving with the same rotatory velocity about an axis similarly placed, will likerwise be in equilibrio, supposing that its particles attract one another by the same law.

The second principle is susceptible of a very clear derronstration, to which no objection can be made. If we take any two particles placed alike in the two bodies, the attractive and centrifugal forces will have the same invariable proportion; namely, that of the linear dimensions of the bodies. Thus the forces that act upon the particles of one body, are all augmented, or all diminished, in the same constant ratio in the other body; wherefore if there be an equilibrium in one case, there must likewise be an equilibrium in the other case. Hence it is easy to deduce, that when a mass of a homogeneous fluid is in equilibrio, all the level surfaces must be similar to one another; and this is sufficient to determine a priori the figure which the body will assume.

With regard to the first principle, I reasoned in the manner following. The pressure which any exterior level stratum exerts upon the fluid below it, must be perpendicular to the separating surface. But this pressure is the effect of two distinct causes. One is the combined effect of the attraction of the interior matter and the centrifugal force, which two forces constitute the gravitation at the separating surface; the other 
is the attraction of the stratum upon the interior matter. Now it is a mathematical property of the level surfaces, that the gravitation at any such surface causes an exterior level stratum in contact with it to press perpendicularly upon the interior fluid; whence it follows that the attraction of the exterior matter upon the interior fluid must likewise cause a pressure perpendicular to the separating surface. The interior fluirl must therefore be in equilibrio by the attraction of the exterior stratum; which can happen only beciause every particle of the interior fluid is urged equally in opposite directions by the attraction of the stratum. It will be allowed that the equilibrium in question is a necessary consequence of the cause assigned; and when we consider that any particle within the stratum, is on every side urged outward by the attraction, it seems impossible not to admit the soundness of the principle. It must be recollected that, in all this, we are here speaking only of a homogeneous fluid; and we may even confine our attention to the equilibrium of such a fluid acted upon by the attraction of its particles in the inverse proportion of the square of the distance and by a centrifugal force. In this view there is no doubt of the truth of the first principle, for it is easily deduced from the second. Either of them is sufficient to determine the figure of equilibrium a priori, and they prove that it can only be an oblate elliptical spheroid.

It is a great mistake to suppose that it is intended to advance any thing in opposition to the general equation of equilibrium, in which all the forces in action are taken into account. But that general equation will not apply to particular problems without some modifications which it is necessary to investigate.

It is of great consequence to deternine the figure of a homogeneous fluid in equilibrio, because that of a heterogeneous mass will depend upon it, and must be deduced from it. In a body in equilibrio, which consists of strata of variable density, it is obvious that very near the centre we may consider the density as uniform or constant. This small central body of constant density, being in equilibrio by the attractions of its particles and a centrifugal force, will therefore have the figure of an oblate elliptical spheroid. It is obvious that we may suppose it to become hard and solid, and then it will form a nucleus upon which all the superior strata will press and rest. Supposing now that the nucleus is given, we must determine the figure necessary to the equilibrium of every one of the superior strata; which requires that a proper form of expression be given to the radius of every stratum. Let $\phi^{(2)}$, $\varphi^{(4)}, \varphi^{(6)}, 8 c$. denote functions of $2,4,6$, \&c. dimensions of Vol. 67. No.398. June 1826. 
three rectangular coordinates of a sphere, that is, of the quantities,

$$
\mu, \sqrt{1-\mu^{2}} \cos \pi, \sqrt{1-\mu^{2}} \sin \sigma ;
$$

then, $r$ being the radins of a stratum, we shall have,

$$
r=a\left\{\mathrm{I}+e \varphi^{(z)}+e^{z} \varphi^{(4)}+e^{3} \varphi^{(6)}+\& \mathrm{c} .\right\},
$$

$e$ being a quantity according to which the steps of approximation are to be arranged. This general expression supposes that the central nucleus is an ellipsoid; but if it be a spheroid of revolution, then we must make $\varphi^{(2)}$ equal to $\mu^{2}$, or $1-\mu^{2}$; and $\dot{\phi}^{(4)}, \phi^{(6)} \& c$. must be trinomial, quadrinomial, \&c. functions of $\mu^{2}$. The quantity $e$, the density of the stratum, and the coefficients of all the angular quantities are functions of $a$; and they must be determined so that a stratum shall satisfy the conditions of equilibrium, and, when it comes to the centre, shall rest upon the solid nucleus. In this manner we shall have the most general solution of the equilibrium of a heterogeneous mass of fluid, pushed to any proposed degree of approximation. I am, \&c.

June 14, 1826.

J. IroRy.

LXIV. Supplement to Mr. Henapatro's Paper in the Philosophical Magazine for August 1825, on Functional Equations. By John Herapath, Esq.

To the Editor of the Philosophical Magazine and Journal. Sir,

FROM the period of sending my last communication to the Phil. Mag., my mind has been so much estranged from the consideration of functions, that I might almost be said to have entirely abandoned them. This is the reason why so long a time has elapsed without my having noticed the omission of a restriction, which, on reperusing the paper alluded to a few days since, I was surprised to find I have no where distinctly mentioned. It should have been stated, that the arguments of the paper relate to those periodical functional equations only, rohose solutions contain arbitrary functions. We may indeed understand this from "arbitrary functions," " complete solutions," \&c. in the heading of the paper; but as there are an infinite number of periodical functional equations, having their solutions complete, without containing arbitrary functions, and therefore wanting the properties which render our reasoning applicable, I hasten to supply the omission. 